\title{
An Adaptive MIMO-OFDMA Relay System
}

\author{
Daniel Munoz, Student Member, IEEE, Baile Xie, Student Member, IEEE, \\ and Hlaing Minn, Senior Member, IEEE
}

\begin{abstract}
We consider multi-input multi-output (MIMO) antennas relay systems under time-varying channels and propose a joint relay, antenna, subband, and MIMO transmission mode adaptation scheme which enables antenna selection to exploit channel state information (CSI) at the beginning of transmission and switch to space-frequency block coding (SFBC) when CSI becomes outdated. Moreover, we propose temporal power and bit loading for the cascaded source-relay-destination link with/without inter-hop power allocation to further enhance the system performance.
\end{abstract}

Index Terms-Relay, time-varying, adaptive transmission.

\section{INTRODUCTION}

$\mathbf{R}$ ELAY systems offer effective coverage range extension, and recent studies [1]-[12] extend to systems with multiple potential relay nodes. Both amplify and forward (AF) [1][7] and decode and forward (DF) [6]-[12] relaying strategies are studied, and recently MIMO antenna systems are incorporated [1], [2], [8]-[10]. Most of these studies [1]-[3], [8]-[12] focus on single carrier systems while some [4]-[7] address orthogonal frequency division multiplexing/multiple access (OFDM/OFDMA) with relay selection which requires CSI, and hence entails CSI acquisition. In practical time-varying channels, the CSI acquisition overhead grows with mobile speed; the CSI delay and gradual degradation of CSI within a transmission frame occur which will inevitably affect the existing relaying strategies which assume quasi-static channels during the transmission frame. For non-relay systems, an adaptive transmission using beamforming and space-frequency block coding (SFBC) has been recently proposed in [13]. But for relay systems, a new relay transmission scheme to combat the CSI degradation issue in time-varying channels is needed.

In this paper, we propose an adaptive relay transmission scheme for MIMO-OFDMA with multiple relay nodes in timevarying channels. Our approach is built upon the joint relay and antenna selection (JRAS) scheme from [8] which applies transmit antenna selection (TAS) and receive maximum ratio combination (MRC) in single carrier systems with quasistatic channels and no CSI delays. We extend it to OFDMA systems in time-varying channels with CSI delays, incorporate subband selection, and more importantly introduce a new transmission scheme which selects TAS or SFBC for each OFDM symbol of each hop (source to relay or relay to destination). The MIMO mode selection is based on bit error rate (BER) conditioned on the CSI, the CSI delay, and the channel temporal correlation. TAS offers an advantage when

Manuscript received May 10, 2012. The associate editor coordinating the review of this letter and approving it for publication was F. Gao.

The authors are with the Department of Electrical Engineering, The University of Texas at Dallas (e-mail: \{dan.munoz, baile.xie, hlaing.minn\}@utdallas.edu).

Digital Object Identifier 10.1109/WCL.2012.071612.120357
CSI is fresh while SFBC yields better performance when CSI becomes outdated. Our proposed adaptive scheme absorbs advantages of both TAS and SFBC and hence offers consistently better performance than the two schemes. In addition, we propose adaptive temporal power and bit loading for the cascaded source-relay-destination link with/without inter-hop power allocation to further exploit the CSI and provide greater improvement in both system reliability and capacity.

\section{System Model}

We consider a DF relaying system with a single source, single destination, and $N_{R} \geq 1$ relays. Let $I_{i}$ represent the $i$ th among $M_{I}$ antennas of node $I$, which can be a source node $(S)$, relay node $k\left(R_{k}\right)$ or a destination node $(D)$. The channel has a log-normal shadowing fading with the log mean $\mu_{\mathrm{dB}}$ and variance $\sigma_{\mathrm{dB}}^{2}$ and a Rayleigh distribution for small scale fading. The channel is approximately static within an OFDM symbol but varies within a frame of $K$ OFDM symbols. The maximum number of sample-spaced channel taps is $L$ and the cyclic prefix length is $N_{\mathrm{CP}}>L$. Let $\mathbf{g}_{S_{i} R_{k j}}^{(n)} \in \mathcal{C}^{L \times 1}$ be the time-domain low-pass equivalent channel vector (including both shadowing and small scale fading effects) between $S_{i}$ and $R_{k j}$ at symbol time $n=1,2, \cdots, K$; we have

$$
\mathbf{g}_{S_{i} R_{k j}}^{(n)}=\rho_{n} \mathbf{g}_{S_{i} R_{k j}}^{(0)}+\psi_{S R_{k}} \sqrt{1-\rho_{n}^{2}} \mathbf{w}_{S_{i} R_{k j}}^{(n)},
$$

where $\psi_{S R_{k}}$ is the shadowing factor between $S$ and $R_{k}$; $\mathbf{g}_{S_{i} R_{k j}}^{(0)}$ represents the available CSI at the beginning of a frame (and it may already be outdated to a certain degree). We assume the channel taps are independently distributed. $\rho_{n}$ is the time correlation coefficient between the initial CSI $\mathbf{g}_{S_{i} R_{k j}}^{(0)}$ and the current channel realization at symbol time $n . \mathbf{w}_{S_{i} R_{k j}}^{(n)} \in \mathcal{C}^{L \times 1}$ is a perturbation vector which can represent not only the channel variations but also errors due to channel estimation and quantization for CSI feedback. The entries of $\mathbf{w}_{S_{i} R_{k j}}^{(n)}$ are independent and identically distributed (i.i.d.) complex Gaussian random variables with zero mean and unit variance but the same entries at different $n$ values are correlated according to the channel temporal correlation. Taking discrete Fourier transform (DFT) of (1), we have the equivalent frequency-domain channel at symbol time $n$ as

$$
\mathbf{c}_{S_{i} R_{k j}}^{(n)}=\rho_{n} \mathbf{c}_{S_{i} R_{k j}}^{(0)}+\psi_{S R_{k}} \sqrt{1-\rho_{n}^{2}} \omega_{S_{i} R_{k j}}^{(n)},
$$

where $\mathbf{c}_{S_{i} R_{k j}}^{(k)} \in \mathcal{C}^{N \times 1}$ and $\omega_{S_{i} R_{k j}}^{(k)} \in \mathcal{C}^{N \times 1}$ correspond to the $N$-point DFT of $\mathbf{g}_{S_{i} R_{k j}}^{(k)}$ and $\mathbf{w}_{S_{i} R_{k j}}^{(k)}$, respectively. The system uses OFDMA and divides $N_{\text {used }}$ totally used subcarriers into $N_{\text {sub }}$ subbands such that each subband has $N_{\text {car }}=N_{\text {used }} / N_{\text {sub }}$ contiguous subcarriers and their channel gains are highly correlated. The channel gain of one subband can be represented by the average of those of $N_{\text {car }}$ subcarriers. 
As a result, we can stack the frequency-domain MIMO channel between $S$ and $R_{k}$ associated with subband $m$ at symbol $n$ in a compact form as $\mathbf{H}_{S R_{k}}^{m, n} \in \mathcal{C}^{M_{R_{k}} \times M_{S}}$ :

$$
\mathbf{H}_{S R_{k}}^{m, n}=\rho_{n} \mathbf{H}_{S R_{k}}^{m, 0}+\psi_{S R_{k}} \sqrt{1-\rho_{n}^{2}} \mathbf{W}_{S R_{k}}^{m, n} .
$$

Assuming no spatial correlation among the MIMO channel $\mathbf{H}_{S R_{k}}^{(n)}$ and the perturbation matrix $\mathbf{W}_{S R_{k}}^{(n)}$, their entries are independent. But across time $n$, they are correlated. Each user can choose a subband from $N_{\text {av }}$ available subbands $\left[m_{1}, m_{2}, \cdots, m_{N_{\mathrm{av}}}\right]$. The selected subband can be different between two hops if subband mapping is allowed.

Note that similar model and notations apply to the channel between relay $R_{k}$ and destination $D$, i.e., $\mathbf{H}_{R_{k}, D}^{(n)}$, as well.

\section{PROPOSED SCHEME}

JRAS [8] can effectively exploit CSI with global CSI at the destination. The destination performs the selection and informs the source and relays. In practice, the performance of JRAS degrades as the channels vary, especially considering the delay of CSI acquisition. Let $\Delta_{S}$ be the delay between the CSI acquisition of the source to relay channels and the start of frame transmission from the source, while $\Delta_{R}$ be the delay between the CSI acquisition of the relay to destination channels and the start of frame transmission from the relay. Typically, $\Delta_{R}$ is greater than $\Delta_{S}$ because the relay needs to decode the entire frame before starting the subsequent transmission to the destination. The presence of the large delay leads to outdated CSI and results in performance degradation of JRAS in time-varying channels.

To exploit the CSI and combat time-varying channels, we include SFBC as an alternative due to its relatively consistent performance over time-varying channels. The idea of our scheme is to employ either TAS or SFBC based on their conditional BERs while incorporating CSI delays. For the $n$th OFDM symbol, we choose the transmission mode $\mathcal{M}^{\star}(n)$ as

$$
\mathcal{M}^{\star}(n)=\arg \min _{\mathrm{TAS}, \mathrm{SFBC}}\left\{P_{n \mid \gamma^{(0)}}^{\mathrm{TAS}}, P_{n \mid \gamma^{(0)}}^{\mathrm{SFBC}}\right\}
$$

where $P_{n \mid \gamma^{(0)}}^{\mathrm{TAS}}$ and $P_{n \mid \gamma^{(0)}}^{\mathrm{SFBC}}$ are the conditional BER of the $n$th OFDM symbol using TAS and SFBC, respectively, given $\gamma^{(0)}$ which is the corresponding effective signal to noise ratio (SNR) based on the initial CSI. Specifically, our proposed scheme utilizes the initial CSI to first select the relay and keeps it fixed until next CSI update. The destination compares the conditional BER between using TAS and SFBC for each symbol of each hop associated with the opted relay, and then chooses the transmission mode with the smaller BER for each symbol of each hop respectively. The selections of the relay and transmission modes are then fed back by the destination to the source and relays. Detailed procedures are as follows.

For relay selection, we employ TAS at the transmitter and MRC at the receiver of both hops. The SNR of the first hop from the $i$ th antenna of $S$ to $R_{k}$ on the $m$ th subband at symbol time $n$ is given by

$$
\gamma_{S_{i}, R_{k}}^{m, n}=\eta_{\mathrm{TAS}}\left\|\mathbf{h}_{S_{i} R_{k}}^{m, n}\right\|^{2}
$$

where $\quad \mathbf{h}_{S_{i} R_{k}}^{m, n}=\left[h_{S_{i} R_{k, 1}}^{m, n}, \cdots, h_{S_{i} R_{k, M_{R}}}^{m, n}\right]^{T}$ is the $i$ th column of $\mathbf{H}_{S R_{k}}^{m, n} ; \eta_{\mathrm{TAS}} \triangleq \frac{E_{S}}{N_{0}}$ where $E_{S}$ is the average transmit energy on a data subcarrier of an OFDM symbol at $S$ and $N_{0}$ is the noise power density. For relay $R_{k}$, we choose the best subband and the antenna of $S$ which corresponds to the maximum instantaneous SNR based on the initial CSI: $\left[m_{S_{i^{\star}}}^{\star}, i_{S, k}^{\star}\right]=$ $\arg \max _{m \in\left\{m_{1}, m_{2}, \cdots, m_{N_{\mathrm{av}}}\right\}} \max _{i \in\left\{1, \cdots, M_{S}\right\}} \gamma_{S_{i}, R_{k}}^{m, 0}$.

Similarly, for the second hop, we have $\left[m_{R_{k}}^{\star}, j_{R_{k}}^{\star}\right]=$ $\arg \max _{m \in\left\{m_{1}, m_{2}, \cdots, m_{N_{\text {av }}}\right\}} \max _{j \in\left\{1, \cdots, M_{R_{k}}\right\}} \gamma_{R_{k, j}, D}^{m, 0}$, where $\gamma_{R_{k}, D}^{m, 0}$ is the SNR of link from the $j$ th transmit antenna at $R_{k}$ to $D$ according to initial CSI. It is defined similarly to (5) except that $\eta_{\mathrm{TAS}}$ should be substituted with $\eta_{\mathrm{TAS}}^{\prime} \triangleq \frac{E_{R}}{N_{0}}$ where $E_{R}$ is the transmit energy at the relay.

The BER of $M$-QAM signal in an additive white Gaussian noise (AWGN) channel with a SNR of $\gamma$ is given as [15] $P(\gamma) \cong \Phi_{M} \sum_{i=1}^{\sqrt{M} / 2} \int_{0}^{\pi / 2} \exp \left\{-\frac{3(2 i-1)^{2} \gamma}{2(M-1) \sin ^{2} \phi}\right\} d \phi$, where $\Phi_{M}=\frac{\sqrt{M}-1}{\sqrt{M}} \frac{4}{\pi \log _{2} M}$. With $\gamma^{(0)}$ as the SNR corresponding to CSI, the conditional BER for symbol $n$ is

$$
\begin{aligned}
P_{n \mid \gamma^{(0)}} & =\int_{0}^{\infty} P(\gamma) f_{\gamma \mid \gamma^{(0)}}(\gamma) d \gamma \\
& =\Phi_{M} \sum_{i=1}^{\sqrt{M} / 2} \int_{0}^{\pi / 2} \Theta\left(-\frac{3(2 i-1)^{2}}{2(M-1) \sin ^{2} \phi}\right) d \phi
\end{aligned}
$$

where $\Theta(t)$ is the moment generating function (MGF) of $\gamma^{(n)}$ given $\gamma^{(0)}$. Provided the channel model in (3), we have $\mathbf{h}_{S_{i \star n}^{\star}, R_{k}}^{m^{\star}} \sim \mathcal{C N}\left(\rho_{n} \mathbf{h}_{S_{i \star}^{\star} R_{k}}^{m^{\star}, 0}, \psi_{S R_{k}}^{2}\left(1-\rho_{n}^{2}\right) \mathbf{I}\right)$ conditioned on $\mathbf{h}_{S_{i^{\star}} m^{\star}, R_{k}}^{m_{k}}$. (The subscript of $m^{\star}$ is omitted for clarity when it appears as superscript but it can be deduced from the subscript of $\mathbf{h}$.) The MGF of $\gamma_{S_{i} \star R_{k}}^{m^{\star}, n}$ can be derived as [13]

$$
\begin{aligned}
& \Theta\left(t, n, \psi_{S R_{k}}, \gamma_{S_{i^{\star}}, R_{k}}^{m^{\star}, 0}\right)=\left(1-t \psi_{S R_{k}}^{2} \eta_{\mathrm{TAS}}\left(1-\rho_{n}^{2}\right)\right)^{-M_{R_{k}}} \\
& \quad \times \exp \left\{\frac{t \eta_{\mathrm{TAS}} \rho_{n}^{2}\left\|\mathbf{h}_{S_{i^{\star}} R_{k}}^{m^{\star}, 0}\right\|^{2}}{\left(1-t \psi_{S R_{k}}^{2} \eta_{\mathrm{TAS}}\left(1-\rho_{n}^{2}\right)\right)}\right\} .
\end{aligned}
$$

Substituting (7) into (6) with $t=-\frac{3(2 i-1)^{2}}{2(M-1) \sin ^{2} \phi}$, the conditional BER of TAS for symbol $n$ is

$$
\begin{aligned}
& P_{n \mid \gamma_{S_{i^{\star}, R_{k}}}^{m^{\star}, 0}}^{\mathrm{TAS}}=\sum_{i=1}^{\sqrt{M} / 2} \int_{0}^{\pi / 2}\left(1-t \psi_{S R_{k}}^{2} \eta_{\mathrm{TAS}}\left(1-\rho_{n}^{2}\right)\right)^{-M_{R_{k}}} \\
& \quad \times \Phi_{M} \exp \left\{\frac{t \eta_{\mathrm{TAS}} \rho_{n}^{2}\left\|\mathbf{h}_{S_{i^{\star}} R_{k}}^{m^{\star}, 0}\right\|^{2}}{\left(1-t \psi_{S R_{k}}^{2} \eta_{\mathrm{TAS}}\left(1-\rho_{n}^{2}\right)\right)}\right\} d \phi .
\end{aligned}
$$

On the other hand, for each hop, we can also employ orthogonal SFBC for data transmission. The effective SNR using SFBC from $S$ to $R_{k}$ at symbol time $n$ is

$$
\gamma_{S R_{k}}^{m, n}=\frac{a E_{S}}{M_{S}}\left\|\mathbf{H}_{S R_{k}}^{m, n}\right\|_{F}^{2}=\eta_{\mathrm{SFBC}}\left\|\tilde{\mathbf{h}}_{S R_{k}}^{m, n}\right\|^{2}
$$

where $\eta_{\mathrm{SFBC}} \triangleq \frac{a E_{S}}{M_{S} N_{0}}$ with $a$ as a parameter associated with SFBC code (for Alamouti code, $a=1$ and for rate $1 / 2$ code with 4 transmit antennas, $a=2)$, and $\tilde{\mathbf{h}}_{S R_{k}}^{m, n} \triangleq \operatorname{vec}\left(\mathbf{H}_{S R_{k}}^{m, n}\right)$. Following the same analysis of TAS, we choose the subband at the source as $m_{S R_{k}}^{\star}=\arg \max _{m \in\left\{m_{1}, m_{2}, \cdots, m_{N_{\mathrm{av}}}\right\}} \gamma_{S R_{k}}^{m, 0}$ and the conditional BER using SFBC reads as

$P_{n \mid \gamma_{S R_{k}}^{m^{\star}, 0}}^{\mathrm{SFBC}}=\sum_{i=1}^{\sqrt{M} / 2} \int_{0}^{\pi / 2}\left(1-t \psi_{S R_{k}}^{2} \eta_{\mathrm{SFBC}}\left(1-\rho_{n}^{2}\right)\right)^{-M_{S} M_{R_{k}}}$ 


$$
\times \Phi_{M} \exp \left\{\frac{t \eta_{\mathrm{SFBC}} \rho_{n}^{2}\left\|\tilde{\mathbf{h}}_{S R_{k}}^{m^{\star}, 0}\right\|^{2}}{\left(1-t \psi_{S R_{k}}^{2} \eta_{\mathrm{SFBC}}\left(1-\rho_{n}^{2}\right)\right)}\right\} d \phi .
$$

Similarly, $\gamma_{R_{k} D}^{m^{\star}, 0}$ and $P_{n \mid \gamma_{R_{k} D}^{m \star n}}^{\mathrm{SFBC}}$, the SNR and conditional BER of the link $R_{k}$ to $D$ are given as in (9) and (10), respectively, except with $\tilde{\mathbf{h}}_{S R_{k}}^{m^{\star}, 0}, \psi_{R_{k} D}, m_{R_{k} D}^{\star}$ and $\eta_{\mathrm{SFBC}}^{\prime}=\frac{a E_{R}}{M_{R_{k} N_{0}}}$.

For each link associated with relay $R_{k}$, one can choose a mode between TAS and SFBC, which returns a smaller conditional BER based on CSI of the middle OFDM symbol within

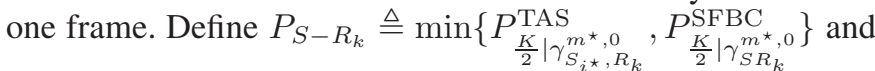
$P_{R_{k}-D} \triangleq \min \left\{P_{\frac{K}{2} \mid \gamma_{R_{k_{j}^{*}, D}}^{\mathrm{TAS}}}^{\mathrm{TA}}, P_{\frac{K}{2} \mid \gamma_{R_{k} D}^{m \star, 0}}^{\mathrm{SFBC}}\right\}$. The best relay $k^{\star}$ can be chosen based on a min-max criterion:

$$
k^{\star}=\underset{k=1, \ldots, N_{R}}{\arg \min } \max \left[P_{S-R_{k}}, P_{R_{k}-D}\right] .
$$

In the remaining, we keep the selected relay $R_{k^{\star}}$, transmit antenna $i_{S, k^{\star}}^{\star}$ of $S$ and $j_{R_{k^{\star}}}^{\star}$ of $R_{k^{\star}}$, as well as the associated subbands fixed. Substituting them into (8) and (10), we obtain the conditional BERs of $n$th OFDM symbol using TAS and SFBC respectively and then adapt the transmission mode, as proposed in (4). This adaptation also applies to the second hop based on its corresponding CSI delay.

To reduce the complexity, an approximate BER expression of $M$-QAM in AWGN channel [14]: $P(\gamma) \approx 0.2 \exp \left\{-\xi_{M} \gamma\right\}$ where $\xi_{M}=\frac{3}{2(M-1)}$ for square $M-\mathrm{QAM}$ and $\xi_{M}=\frac{6}{5 M-4}$ for rectangular $M$-QAM, can be used to obtain closed-form conditional BERs as $P_{n \mid \gamma(0)}=0.2 \Theta\left(-\xi_{M} \gamma\right)$, instead of the finite integration in (8) and (10), In particular, for TAS and SFBC, respectively, we have

$$
\begin{aligned}
& P_{n \mid \gamma_{S_{i^{\star}}, R_{k^{\star}}}^{m^{\star}, 0}}^{\mathrm{TAS}}=0.2\left(1+\xi_{M} \psi_{S R_{k^{\star}}}^{2} \eta_{\mathrm{TAS}}\left(1-\rho_{n}^{2}\right)\right)^{-M_{R_{k^{\star}}}} \\
& \times \exp \left\{\frac{-\xi_{M} \eta_{\mathrm{TAS}} \rho_{n}^{2}\left\|\mathbf{h}_{S_{i^{\star}} R_{k^{\star}}}^{m^{\star}, 0}\right\|^{2}}{1+\xi_{M} \psi_{S R_{k^{\star}}}^{2} \eta_{\mathrm{TAS}}\left(1-\rho_{n}^{2}\right)}\right\}, \\
& P_{n \mid \gamma_{S R_{k^{\star}}}^{m *, 0}}^{\mathrm{SFBC}}=0.2\left(1+\xi_{M} \psi_{S R_{k^{\star}}}^{2} \eta_{\mathrm{SFBC}}\left(1-\rho_{n}^{2}\right)\right)^{-M_{S} M_{R_{k^{\star}}}} \\
& \times \exp \left\{\frac{-\xi_{M} \eta_{\mathrm{SFBC}} \rho_{n}^{2}\left\|\tilde{\mathbf{h}}_{S R_{k^{\star}} m^{\star}, 0}^{2}\right\|^{2}}{1+\xi_{M} \psi_{S R_{k^{\star}}}^{2} \eta_{\mathrm{SFBC}}\left(1-\rho_{n}^{2}\right)}\right\} .
\end{aligned}
$$

As we will show later, the adaptive scheme based on (12) and (13) gives similar performance to that using (8) and (10), hence it is preferable due to its computational efficiency.

\section{AdAptive Power And Bit LoAding}

After determining the adaptive MIMO mode, one can further enhance the system reliability via power loading. We use the sum BER metric (i.e., $P^{[1]}+P^{[2]}$ where $P^{[i]}$ is the BER of the hop $i$ ) as it well approximates the effective BER of the cascaded relay system (i.e., $P^{[1]}+P^{[2]}-P^{[1]} P^{[2]}$ ). For successful information transmission from $S$ to $D$, the total energy consumed over the overall link is a valid metric. We can properly assign energies to the two hops under a total energy constraint to minimize the sum BER:

$$
\begin{gathered}
\left\{E_{S}^{\star}, E_{R}^{\star}\right\}=\arg \min P^{[1]}\left(E_{S}\right)+P^{[2]}\left(E_{R}\right) \\
\text { s.t. } E_{S}+E_{R}=\text { Constant, }
\end{gathered}
$$

where $P^{[1]}\left(E_{S}\right)$ is the conditional BER of the middle $(K / 2$ th) symbol of the first hop using (12) or (13) depending on transmission mode $\mathcal{M}^{\star}\left(\Delta_{S}+K / 2\right)$ determined by (4) with energy $E_{S} . P^{[1]}\left(E_{S}\right)$ includes the effect of CSI delay $\Delta_{S}$. $P^{[2]}\left(E_{R}\right)$ is defined similarly for the second hop. This interhop power allocation can be omitted if each hop prefers to use its individual power constraint only.

Temporal power allocation and bit loading can be integrated with our proposed adaptive scheme to improve system capacity while meeting a target error performance. Temporal power allocation usually requires the probability density function of the equivalent channel gain. However, its analytical expression is hardly tractable in our case due to multiple selections performed by the adaptive scheme. Alternatively, we track the empirical distribution of the selected initial equivalent channel gain: $\left\|\mathbf{h}_{0}\right\|^{2} \triangleq \min$ $\left[\left\|\mathbf{h}_{S_{i^{\star}} R_{k^{\star}}, 0}^{m^{\star}, R^{2}}, \frac{a}{M_{S}}\right\| \tilde{\mathbf{h}}_{S R_{k^{\star}} m^{\star}, 0}^{m^{*}}\left\|^{2},\right\| \mathbf{h}_{R_{k_{j}^{\star}}}^{m^{\star}, 0}\left\|^{2}, \frac{a}{M_{S}}\right\| \tilde{\mathbf{h}}_{R_{k^{\star}} m^{\star} D}^{m^{\star}, 0} \|^{2}\right] \quad$ offline. By evenly dividing the range of $\left\|\mathbf{h}_{0}\right\|^{2}$ into $q$ bins (except the last one ranging up to $\infty$ ), we then calculate the average values and probability of $\left\|\mathbf{h}_{0}\right\|^{2}$ falls into each bin, based on which, the classic water-filling algorithm of power loading for capacity maximization is performed and return a power level vector. Thus, for each channel realization, we only need to map the instantaneous $\left\|\mathbf{h}_{0}\right\|^{2}$ into one of the $q$ bins and use the corresponding pre-computed power level $E_{\mathrm{tmp}}$, yielding very low complexity implementation. Given a target error performance $P_{\mathrm{tar}}$, one can adjust modulation size $M$ to ensure both hops' BERs $P_{S-R_{k^{\star}}}$ and $P_{R_{k^{\star}}-D}$ based on $\left\|\mathbf{h}_{0}\right\|^{2}$ and the assigned power $E_{\mathrm{tmp}}$ are below $P_{\mathrm{tar}}{ }^{1}$.

\section{Simulation Results and Discussions}

The same parameters are set for all results below: the carrier frequency of $1.92 \mathrm{GHz}$, subcarrier spacing of $15 \mathrm{kHz}$, $N=64$ subcarriers, $N_{\mathrm{CP}}=16$ and $K=14$ OFDM symbols per frame. Two antennas are used at each node for either transmission or reception. Rayleigh fading channel is assumed with $L=4$ sample-spaced uncorrelated taps and a normalized $3-\mathrm{dB}$ per tap decaying power delay profile. The log-mean and variance are $\mu_{\mathrm{dB}}=0$ and $\sigma_{\mathrm{dB}}^{2}=4$ for the shadowing effects. The delays are $\Delta_{S}=5$ and $\Delta_{R}=K+4$ OFDM symbols.

First we show the average BERs of $K$ OFDM symbols on the source to relay hop with $N_{\mathrm{av}}=1$ and $\frac{E_{S}}{N_{0}}=\frac{E_{R}}{N_{0}}=$ $6 \mathrm{~dB}$ in Fig. 1. The analytical expressions in (8) and (10) reflects the simulation results better than those in (12) and (13). However, both yield essentially the same performance for the MIMO mode adaptation. The adaptive scheme chooses TAS over SFBC for the first few symbols because of its better CSI exploitation. As the delay increases, TAS loses advantage to SFBC due to outdated CSI within one frame. Our adaptive scheme obtains a better BER than both of the two alternatives.

Fig. 2 shows the overall BERs of the relay system at various SNR values (SNR is defined as $\left(E_{S}+E_{R}\right) /\left(2 N_{0}\right)$ ) with mobile speed $v_{1}=30 \mathrm{~km} / \mathrm{h}$ and $v_{2}=40 \mathrm{~km} / \mathrm{h}$ in the first and second hops. If the user chooses from $N_{\mathrm{av}}=4$ subbands and allows different subbands used for the two

\footnotetext{
${ }^{1}$ This corresponds to a pessimistic design due to the worst-case BER considered using $\left\|\mathbf{h}_{0}\right\|^{2}$. We can appropriately loosen the BER threshold in determining $M$ while the actual error performance still meets the requirement.
} 


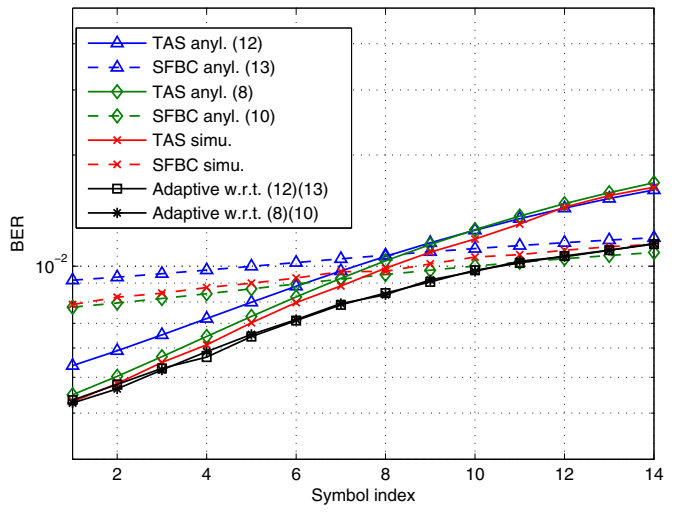

Fig. 1. Comparison of the adaptive scheme with TAS and SFBC in the source to relay hop with $v_{1}=v_{2}=80 \mathrm{~km} / \mathrm{h}$ and $E_{S} / N_{0}=E_{R} / N_{0}=6 \mathrm{~dB}$.

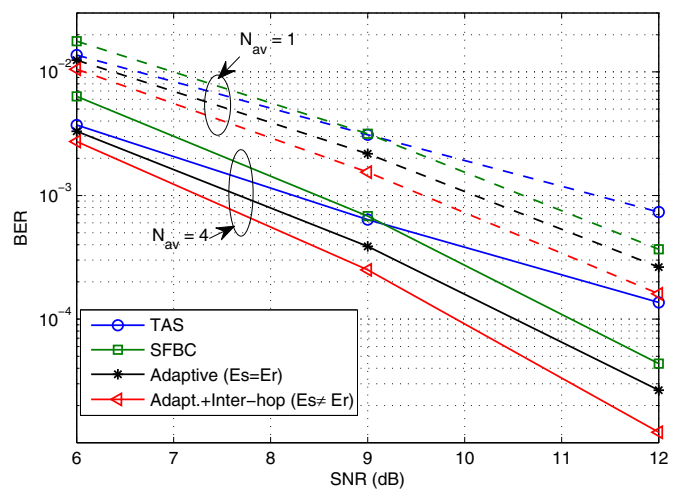

Fig. 2. Comparison of the adaptive scheme with/without power allocation versus TAS and SFBC for the overall relay system with $v_{1}=30 \mathrm{~km} / \mathrm{h}, v_{2}=$ $40 \mathrm{~km} / \mathrm{h}$ and QPSK.

hops, about 2 SNR dB gain is observed for all schemes due to the diversity gain compared with those without subband selection $\left(N_{\mathrm{av}}=1\right)$. Despite the value of $N_{\mathrm{av}}$, our adaptive scheme always outperforms both TAS and SFBC for the entire SNR range. Moreover, with the proposed inter-hop power allocation, an additional performance enhancement can be achieved. For example, at least $1.5 \mathrm{~dB}$ SNR gain compared to pure TAS or SFBC at BER of $5 \times 10^{-3}$ for $N_{\mathrm{av}}=4$ case.

With the fixed-rate results in Fig. 2, given a desired BER of $10^{-3}$, the corresponding SNR points required by each scheme can be found: about $7.7 \mathrm{~dB}, 8.25 \mathrm{~dB}$ and $8.5 \mathrm{~dB}$ for adaptive scheme, TAS and SFBC when $N_{\mathrm{av}}=4$ while 10.1 dB, $10.6 \mathrm{~dB}$ and $11.35 \mathrm{~dB}$ for adaptive scheme, SFBC and TAS when $N_{\mathrm{av}}=1$. By applying the proposed temporal power and bit loading (TPBL) with $q=16$ power levels and $M \in[2,4,8,16,64]$, the system throughput improves compared to the fixed rate cases as shown in Fig. 3 where all points have approximately the same actual BER of $10^{-3}$. Up to $15 \%$ and $25 \%$ bit rate increase can be attained by the adaptive scheme with TPBL and the adaptive scheme with TPBL and inter-hop power loading compared to TAS while $13 \%$ and $20 \%$ increase compared to SFBC, respectively.

\section{CONCLUSION}

We propose a joint relay, antenna, subband, and MIMO transmission mode adaptation scheme for MIMO-OFDMA relay systems, which combines the advantages of both TAS

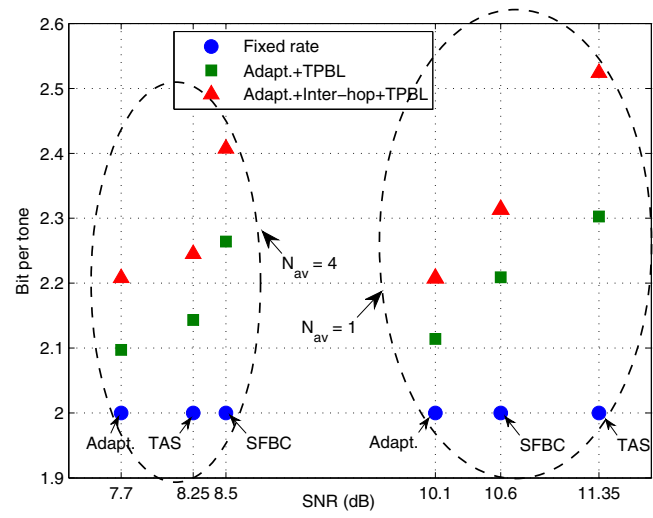

Fig. 3. Throughput improvements of temporal power loading and bit loading (TPBL) for $v_{1}=30 \mathrm{~km} / \mathrm{h}$ and $v_{2}=40 \mathrm{~km} / \mathrm{h}$ at BER of $10^{-3}$.

and SFBC by adaptively switching between the two for a smaller conditional BER based on the initial CSI. Along with the proposed temporal power and bit loading with/without inter-hop power allocation, the proposed method achieves further reliability and throughput enhancements due to better CSI exploitation and greater robustness to channel variations with practical CSI delays.

\section{REFERENCES}

[1] R. Vaze and R. W. Heath Jr., "To code in space and time or not in multihop relay channels," IEEE Trans. Signal Process., vol. 57, no. 7, pp. 2736-2747, July 2009.

[2] S. O. Gharan, A. Bayesteh, and A. K. Khandani, "Diversity-multiplexing tradeoff in multiple-relay network_part II: multiple-antenna networks," in Proc. 2008 IEEE Allerton Conf., pp. 95-102.

[3] Y. Jing and H. Jafarkhani, "Single and multiple relay selection schemes and their achievable diversity orders," IEEE Trans. Wireless Commun., vol. 8, no. 3, pp. 1414-1423, Mar. 2009.

[4] F. Li, X. Ke, and L. Wang, "A relay selection scheme with limit feedback in OFDM relay networks based on subcarrier mapping," IEEE YC-ICT, pp. 93-96, Nov. 2010.

[5] R. Yin, Y. Zhang, G. Yu, Z. Zhang, and J. Zhang, "Distributed joint optimization of relay selection and subchannel pairing in OFDM based relay networks," in Proc. 2009 IEEE PIMRC, pp. 722-726.

[6] W. Lijie, Z. Haitao, Xiyong, and W. Jibo, "Optimizing the performance of OFDM-based cooperative networks by relay selection, power allocation and relay localization," in Proc. 2010 IEEE ICUWB, vol. 2.

[7] L. Wang, H. Zhao, Y. Tang, and J. Wei, "The joint relay selection with power allocation for outage-optimization in OFDM-based cooperative relaying," in Proc. 2010 ICCASM, vol. 8, pp. 557-561.

[8] M. Ju, H. K. Song, and I. M. Kim, "Joint relay-and-antenna selection in multi-antenna relay networks," IEEE Trans. Commun., vol. 58, no. 12, pp. 3417-3422, Dec. 2010.

[9] R. Tannious and A. Nosratinia, "Spectrally-efficient relay selection with limited feedback," IEEE J. Sel. Areas Commun., vol. 26, no. 8, pp. 14191428, Oct. 2008.

[10] T. Zhang, S. Zhao, L. Cuthbert, and Y. Chen, "Energy-efficient cooperative relay selection scheme in MIMO relay cellular networks," in Proc. 2010 IEEE ICCS, pp. 269-273.

[11] V. Shah, N. B. Mehta, and R. Yim, "The relay selection and transmission trade-off in cooperative communication systems," IEEE Trans. Wireless Commun., vol. 9, no. 8, pp. 2505-2515, Aug. 2010.

[12] Q. You, Y. Li, and Z. Chen, "Joint relay selection and network coding using decode-and-forward protocol in two-way relay channels," 2010 IEEE GLOBECOM.

[13] B. Kecicioglu, H. Minn, and C. C. Chong, "Intra-frame transmission adaptation for fast fading MIMO-OFDMA systems," IEEE Trans. Commun., vol. 59, no. 9, pp. 2585-2594, Sep. 2011.

[14] S. Zhou and G. B. Giannakis, "Adaptive modulation for multiantenna transmissions with channel mean feedback," IEEE Trans. Wireless Commun., vol. 3, no. 5, pp. 1626-1636, Sep. 2004.

[15] M. K. Simon and M.-S. Alouini, Digital Communications Over Fading Channels. Wiley, 2000. 